\title{
Understanding the role of the family physician in early psychosis intervention
}

Kelly K. Anderson, Suzanne Archie, Richard G. Booth, Chiachen Cheng, Daniel Lizotte, Arlene G. MacDougall, Ross M. G. Norman, Bridget L. Ryan, Amanda L. Terry and

Rebecca Rodrigues, on behalf of the project co-investigators

\section{Background}

The family physician is key to facilitating access to psychiatric treatment for young people with first-episode psychosis, and this involvement can reduce aversive events in pathways to care. Those who seek help from primary care tend to have longer intervals to psychiatric care, and some people receive ongoing psychiatric treatment from the family physician.

\section{Aims \\ Our objective is to understand the role of the family physician in help-seeking, recognition and ongoing management of} first-episode psychosis.

\section{Method}

We will use a mixed-methods approach, incorporating health administrative data, electronic medical records (EMRs) and qualitative methodologies to study the role of the family physician at three points on the pathway to care. First, help-seeking we will use health administrative data to examine access to a family physician and patterns of primary care use preceding the first diagnosis of psychosis; second, recognition: we will identify first-onset cases of psychosis in health administrative data, and look back at linked EMRs from primary care to define a risk profile for undetected cases; and third, management: we will examine service provision to identified patients through EMR data including patterns of contacts, prescriptions and referrals to specialised care. We will then conduct qualitative interviews and focus groups with key stakeholders to better understand the trends observed in the quantitative data.

\section{Discussion}

These findings will provide an in-depth description of firstepisode psychosis in primary care, informing strategies to build linkages between family physicians and psychiatric services to improve transitions of care during the crucial early stages of psychosis.

\section{Declaration of interest \\ None.}

\section{Keywords}

First-episode psychosis; early intervention; primary care; family physician; pathways to care.

\section{Copyright and usage}

(c) The Royal College of Psychiatrists 2018. This is an Open Access article, distributed under the terms of the Creative Commons Attribution-NonCommercial-NoDerivatives licence (http://creativecommons.org/licenses/by-nc-nd/4.0/), which permits noncommercial re-use, distribution, and reproduction in any medium, provided the original work is unaltered and is properly cited. The written permission of Cambridge University Press must be obtained for commercial re-use or in order to create a derivative work.
Evidence suggests that timely and adequate management of the first episode of psychosis is a clinical imperative. Providing rapid access to psychiatric treatment can prevent unnecessary suffering of patients and their family members, as well as prevent the negative social, educational and occupational consequences associated with untreated psychosis. Evidence from systematic reviews suggest that delays in the treatment of psychosis are associated with poor clinical and functional outcomes, ${ }^{1,2}$ and outcome trajectories are typically determined in the 2-year period following the first episode, ${ }^{3}$ making the early stages of psychotic disorder a critical period for detection and intervention. This evidence has led to the widespread implementation of early psychosis intervention (EPI) services, reflecting optimism about prospects for recovery if comprehensive services are offered early in the course of illness. ${ }^{4}$

The emphasis on early detection and reduction of treatment delay in first-episode psychosis has led to an increased interest in pathways to care. ${ }^{5}$ The Goldberg and Huxley model has been proposed as a framework to describe the process of seeking mental healthcare. This model proposes that there are four 'filters' between five levels of care, and patient characteristics, clinical features, physician attributes and systemic barriers influence whether a person will progress from one level to the next. ${ }^{6}$ The first level of the model is the community, where a considerable number of people suffer from psychiatric symptoms or psychological distress. At the second level are the subset of symptomatic people who seek help from a family physician. ${ }^{8}$ When symptomatic people do seek healthcare, the family physician may only identify psychiatric illness in a subset of patients, which comprises the third level. The fourth level consists of people who are diagnosed as having a psychiatric disorder and are referred to mental health services, and the fifth level includes people who present to mental health services and are subsequently admitted to hospital for more intensive in-patient care. ${ }^{6}$

\section{The family physician and pathways to care}

Research on the pathways to care of young people with first-episode psychosis presents a more complex picture of the types and sequence of contacts than the model proposed by Goldberg and Huxley, with circuitous routes to care, cycling within and between services and heavy use of the emergency department and inpatient admissions. ${ }^{5,9-13}$ Within this complexity, the family physician continues to be a key player in these pathways to mental healthcare. More specifically, approximately $30 \%$ of young people with first-episode psychosis in Ontario (Canada) receive their first diagnosis of psychosis from a family physician. An additional $30 \%$ who were diagnosed in secondary or tertiary care had mental health contacts with a family physician in the 6-month period before the first diagnosis of psychosis. ${ }^{14}$ This indicates that the majority of young people with early psychosis are making helpseeking contacts for mental health problems in primary care.

Additionally, young people with the early signs of psychosis who initiate their own help-seeking (as opposed to family or 
friends on their behalf) are more likely to seek help from a family physician than from psychiatric or emergency services. ${ }^{11}$ This indicates that family physicians may see these young people when they are at an earlier stage of illness and motivated by a subjective need for help, and thus more amenable to engaging in treatment.

Involvement of a family physician on the pathway to care also reduces the likelihood of negative and aversive pathways to care, such as via police, ambulance and the emergency department, ${ }^{9,11,15}$ and reduces the likelihood of subsequent in-patient admission. ${ }^{15}$ Increasing family physician involvement in the identification and management of young people with early psychosis would be beneficial for improving service-related outcomes.

Moreover, young people with first-episode psychosis who seek help from a family physician tend to have a longer period of time before first contact with a psychiatrist and a greater number of help-seeking contacts, relative to young people with psychosis who present to services elsewhere., ${ }^{9,15}$ This may indicate problems with referrals and wait times, or may indicate that family physicians are having difficulty recognising and responding to these patients.

Finally, a substantial proportion of young people with psychosis in Ontario are not accessing EPI services and are receiving ongoing psychiatric treatment in primary care. ${ }^{16}$ We do not have information on the care being provided to these patients - this could indicate gaps in EPI service provision or may suggest that a subset of patients do well in primary care and do not need the more intensive and specialised care offered by EPI programmes.

\section{The family physician and early psychosis}

Although these findings highlight the importance of the family physician in pathways to care, they are unable to provide explanations for the observed trends. Surveyed family physicians estimate that they see one or two patients per year with the signs of early psychosis, ${ }^{17-20}$ and physicians with poor knowledge of the core signs of first-episode psychosis are more likely to report that they did not see any patients in the previous year suspected to be in the early phases of a psychotic disorder. ${ }^{19}$ Many primary care clinicians report lacking confidence in their diagnostic skills for firstepisode psychosis because of the fluctuating nature of symptoms and the high prevalence of subthreshold psychosis in the community. ${ }^{21}$ Indeed, international research using health administrative data has shown increased use of primary care services up to 6 years before a first diagnosis of psychosis. ${ }^{22}$ These help-seeking contacts may have occurred during the putative 'prodromal phase' that precedes first-episode psychosis, which is characterised by a heterogeneous pattern of symptoms, including depressed mood, anxiety, sleep disturbance, social withdrawal, deterioration in functioning and irritability. ${ }^{23}$ When the signs of early psychosis are recognised by family physicians, there may be uncertainty regarding how to proceed, and some report they lack the requisite skills and knowledge for dealing with people with serious mental illness and perceive these patients as too specialised for primary care. $^{24}$ Family physicians report that they rarely initiate antipsychotic treatment in a suspected case of first-episode psychosis, and instead prefer a psychiatric referral or consultation to confirm the diagnosis. ${ }^{17,25}$ Organising a prompt referral to specialised services may not always be straightforward: nearly half of family physicians in a large survey reported only referring suspected cases when the referral is requested or accepted by the patient or when the diagnostic picture becomes clear, ${ }^{17}$ and family physicians report that the delay that arises from convincing reluctant patients to accept a referral can be longer than a month for nearly half of all cases. ${ }^{25}$ Family physicians also report delays obtaining a rapid referral because of inaccessibility of mental health services and a lack of communication with psychiatrists, ${ }^{17,25}$ and even when referrals are successfully initiated, many family physicians report that a substantial number of patients are lost to follow-up between primary and secondary services. ${ }^{26}$ Finally, less than half of young people with psychotic disorders who are receiving care from specialised services have ongoing contact with primary care services. ${ }^{27}$ This concurrent contact is needed to monitor risk factors associated with psychotic disorders and antipsychotic treatment, such as smoking and obesity, and to manage medical comorbidities.

\section{Rationale}

Because first-episode psychosis is a relatively rare occurrence in primary care, the importance of the family physician in early detection and intervention has been underestimated; further, it has been argued that EPI services need to more actively engage with the primary care sector to ensure success. ${ }^{28}$ However, there is a notable lack of literature on patterns of primary care use for young people with first-episode psychosis. Most prior research has used surveys of family physicians, which are unable to provide an accurate picture of clinical activities, service provision and the factors leading to patients who go undiagnosed in the primary care system. Of exception, two prior studies have used health administrative data to look at help-seeking from a family physician before the first diagnosis of psychosis, ${ }^{15,22}$ but health administrative data alone does not yield information on the reasons behind observed trends.

It is evident that there is a need to support family physicians in the important role that they play in the help-seeking process of young people with first-episode psychosis. A number of studies have attempted to intervene at the level of primary care to improve detection and referral rates, and the results of these interventions have been equivocal: some studies, ${ }^{18,29,30}$ but not all, ${ }^{31}$ have found that family physician education increases referrals to secondary care, but other evidence suggests that education alone is unlikely to substantially improve detection and referral rates. ${ }^{32}$ Recent evidence from the UK has found that an intensive liaison between primary and secondary care is both effective and cost-effective for improving detection rates of early psychosis. ${ }^{33}$ To more effectively design and implement initiatives to support family physicians in this role, we need a thorough understanding of the underlying reasons for the trends currently observed in primary care.

\section{Method}

The overall goal of this study is to understand the role of the family physician and primary care services in the help-seeking process, recognition and ongoing management of young people with firstepisode psychosis. This project will use a mixed-methods approach, specifically a sequential explanatory design that uses qualitative data to help explain or expand upon significant or anomalous trends observed in quantitative findings. ${ }^{34}$ For each of the proposed objectives, we will use population-based health administrative data and/ or electronic medical records (EMRs) to provide quantitative information. Following analyses of these data, we will conduct qualitative interviews and focus groups with key stakeholders to better understand the trends we observe in the quantitative data.

\section{Ethics approval and consent to participate}

The Institute for Clinical Evaluative Sciences (ICES) is a prescribed entity under section 45 of Ontario's Personal Health Information Protection Act. Section 45 is the provision that enables analysis and compilation of statistical information related to the management, evaluation and monitoring of, allocation of resources to, 
and planning for the health system. Section 45 authorizes health information custodians to disclose personal health information to a prescribed entity, like ICES, without consent for such purposes. The portions of this protocol that involve health administrative and EMR data do not require review by a research ethics board. Ethics approval for the qualitative portion of the study will be sought in the final year of the project.

\section{Availability of data and materials}

The data-set from this study will be held securely in coded form at ICES and the ICES analyst will have full access to study data. Although data-sharing agreements prohibit ICES from making the data-set publicly available, access can be granted to those who meet prespecified criteria for confidential access, available at www.ices.on.ca/DAS. The full data-set creation plan is available from the authors upon request.

\section{Study objectives}

The objectives addressed in this study will be guided by the first three filters that involve primary care in Goldberg and Huxley's pathways-to-care framework: ${ }^{6}$

(i) Pathway filter 1: help-seeking in primary care. Our first objective is to describe help-seeking from primary care for young people with early psychosis, specifically the proportion of people with first-episode psychosis who have access to a regular family physician, and whether these people have distinct patterns of help-seeking in primary care before a first episode of psychosis.

(ii) Pathway filter 2: recognition by primary care. Our second objective is to identify people with first-episode psychosis who were diagnosed in secondary and tertiary care but had prior help-seeking contacts in primary care, with an aim of identifying a risk profile for prodromal or early cases in primary care.

(iii) Pathway filter 3: management by primary care. Our third objective is to describe the practise patterns and ongoing care provided to young people with first-episode psychosis in primary care.

Following these quantitative analyses, we will conduct a series of qualitative interviews with various stakeholders, to describe (i) the experiences of young people with first-episode psychosis when seeking help from primary care for their early symptoms of psychosis, including facilitators or barriers to help-seeking from a family physician; (ii) the experiences of family physicians in recognising and diagnosing early psychosis in primary care, and any additional supports that may be needed to manage cases of first-episode psychosis in primary care and (iii) the perceptions of clinicians at EPI programmes on barriers and opportunities for providing collaborative care with family physicians.

\section{Source of quantitative data}

Each of the three phases of the study will involve analyses of the data holdings at ICES, which has an extensive repository of linked health administrative data from the publicly funded Ontario Health Insurance Plan (OHIP), with access to data from physician billings, hospital admissions and ambulatory care visits. There are also data derived from the EMRs of more than 350 family physicians from across Ontario, representing over 500000 patients in urban, suburban and rural areas of the province. This database is fully linkable to the health administrative data by encrypted health insurance numbers. The ICES data holdings include the entire population of
Ontario with valid OHIP coverage, with information dating as far back as 1988 .

\section{Pathway filter 1: help-seeking in primary care Research questions}

Our first research question is, what proportion of young people with first-episode psychosis have access to a regular family physician, and what sociodemographic and clinical factors are associated with a lack of access? Our second research question is, do people with first-episode psychosis have distinctive patterns of help-seeking within primary care preceding the first diagnosis, relative to the general population?

\section{Hypotheses}

We hypothesize that young people with first-episode psychosis will be less likely to have access to a regular family physician, and that access will vary by sociodemographic and clinical factors. We also hypothesize that, among people who do have access to a regular family physician, those with first-episode psychosis will demonstrate elevated and increasing help-seeking attempts in the 6-year period preceding the index diagnosis of psychosis, and have a greater proportion of help-seeking attempts for mental health reasons, relative to the general population.

\section{Study design}

We will construct a retrospective cohort composed of Ontario residents aged 14-35 years between 2005 and 2015. This age group is considered a priority population by the Ontario Ministry of Health and Long-Term Care, ${ }^{4}$ and this time period corresponds to the rollout of EPI programmes across the province.

\section{Case status}

New cases of psychotic disorder will be identified by a primary discharge diagnosis of non-affective psychosis (e.g. schizophrenia, schizoaffective disorder, schizophreniform disorder, psychosis not otherwise specified) from a hospital bed, or at least two OHIP billing claims or emergency department visits with a diagnostic code for non-affective psychosis in any 12-month period. This algorithm has been previously validated at ICES, using medical charts. ${ }^{35}$ People with a history of service contact for psychosis before 2005 will be considered prevalent cases and will be removed.

\section{Assignment of family physicians}

Each person in the cohort will be assigned to a family physician with the Client Agency Program Enrolment tables at ICES, which is a database of patients rostered to each family physician practising in a patient enrolment model. For family physicians not practising in these models, a 'virtual roster' method will be used, which assigns non-rostered patients to the family physician who had the highest value of billings for 18 core primary care OHIP fee codes in the previous 2 years. This method has been shown to have high levels of concordance $(>80 \%)$ with self-report measures of access to primary care. ${ }^{36}$ All remaining people will be considered to have no regular family physician.

\section{Covariates}

Available sociodemographic variables include age, gender, income quintile, marginalisation index, rural place of residence, ethnicity and immigrant status. We will also construct indicator variables to reflect the presence of other psychiatric or medical comorbidities that may affect access to primary care, such as history of diagnosis of a substance-related disorder or diabetes. 


\section{Analysis 1}

We will estimate the proportion of young people with newly diagnosed psychosis who do not have regular access to a family physician, and compare this with young people in the general population. We will use multivariate logistic regression to model whether subgroups of young people with psychotic disorder (such as those with rural residence and ethnic minorities) are less likely to have regular access to a family physician.

\section{Analysis 2}

This portion of the analysis will be restricted to young people who are enrolled or virtually rostered with a family physician. We will conduct a case-control analysis, using the population-based control group of young people who do not have a psychotic disorder. Cases will be matched to controls at a ratio of 1:2, based on age $( \pm 1$ year), gender and rural residence at the diagnosis date of the corresponding case. We will compare the pattern of primary care visits between cases and controls for the 6-year preceding period, using negative binomial regression models with robust variance estimators to account for the matched design.

\section{Sample size}

Our previous 10-year cohort of young people aged 14-35 years in Ontario with a first diagnosis of psychotic disorder included over 20000 people, ${ }^{14}$ and our proposed matching procedure will give us a control group of 40000 people. This sample size is more than sufficient to detect small differences in parameter estimates between groups (e.g. rate ratios of 1.1 detected with $100 \%$ power at a $99 \%$ confidence level).

\section{Outputs}

These analyses will allow us to determine whether young people with a first episode of psychotic disorder have differential access to a regular family physician, as has been found among people with chronic psychotic disorders, ${ }^{37}$ and whether this access differs by sociodemographic or clinical factors. It will also enable an exploration of whether young people with psychotic disorders show different patterns of help-seeking from primary care in the period leading up to the first diagnosis, as observed in other jurisdictions. ${ }^{22}$

\section{Pathway filter 2: recognition by primary care \\ Research questions}

Our first research question is, what proportion of young people with first-episode psychosis were diagnosed in secondary and tertiary care but had prior help-seeking contacts in primary care? Our second research question is, can we identify a risk profile for prodromal or early cases in primary care?

\section{Hypotheses}

We hypothesize that patient characteristics such as age and gender, as well as physician characteristics such as graduation year and panel size, will be associated with detection of the early signs of psychosis in primary care. We also hypothesize that a distinct profile of primary care help-seeking will emerge, based on presenting symptoms and other characteristics, that will enable us to identify a risk profile to aid in early detection in the primary care context.

\section{Study design}

We will link the cohort created in the previous analysis to the Electronic Medical Record Administrative Data Linked Database (EMRALD), which includes EMR data from over 350 family physicians. This linkage will allow us to identify cases of first- episode psychosis in the health administrative data, using a validated algorithm, ${ }^{35}$ and then look back in EMR data for more detailed information on prior mental health help-seeking attempts from the family physician. The sample will be restricted to people who are in the EMRALD database with prior contacts in primary care.

\section{Outcome classification}

The sample will be divided into two groups: (i) people who received the index diagnosis of psychosis in primary care and (ii) people who were diagnosed in secondary or tertiary care, but who had prior help-seeking attempts in primary care.

\section{Patient-level covariates}

Available variables include age, gender, income quintile, marginalisation index, rural place of residence, ethnicity, immigrant status and psychiatric and medical comorbidities.

\section{Physician-level covariates}

Available variables include age, gender, years since graduation, rurality of practice, practice model (e.g. fee for service, family health team, etc.) and practice size.

\section{Analysis 1}

Multilevel logistic regression, with physician as the clustering unit, will be used to model the patient and physician characteristics associated with first diagnosis in primary care.

\section{Analysis 2}

We will use machine-learning techniques to define a risk profile for people in primary care who may be experiencing prodromal or early signs of psychotic disorder but go undiagnosed by the family physician. We will use predictive modelling techniques from machine learning (such as decision trees ${ }^{38}$ ), informed by established criterion characterising the prodrome to psychosis, ${ }^{39}$ to look at presenting symptoms and other visit characteristics to identify subgroups of young people who are most likely to receive a subsequent diagnosis of psychosis.

\section{Sample size}

Using a local EMR database, exploratory pilot analyses suggest primary care physicians have a mean of 2.4 young people with early psychosis on their roster. Extrapolating to the EMRALD database, we expect approximately 750 diagnosed cases in primary care. We are unable to determine the number of undiagnosed cases, but prior research suggests that approximately $30 \%$ of young people with first-episode psychosis are diagnosed by a family physician and an additional $30 \%$ had primary care mental health contacts in the 6 months preceding the index diagnosis. ${ }^{14}$ Based on our estimates for the number of diagnosed cases, we anticipate an additional 750 undiagnosed cases in the EMR data, for a total sample of 1500 people. This sample size will allow us to detect odds ratios of $1.2-1.3$, with $80 \%$ power at a $95 \%$ confidence level. This sample size is also sufficient for the machine-learning analyses, where the general criterion is that at least ten cases are needed per variable considered in the decision-tree analysis.

\section{Outputs}

These analyses will allow us to determine whether patient-, physician- or practice-level characteristics are associated with diagnosis of early psychosis in primary care, and to identify a risk profile of early psychosis in primary care to aid family physicians in identification. 


\section{Pathway filter 3: management by primary care}

\section{Research questions}

Our first research question is, how do family physicians manage cases of first-episode psychosis identified in primary care? Our second research question is, what are their practise patterns with respect to prescription of psychotropic medication, referral to specialised services and ongoing management?

\section{Hypotheses}

We hypothesize that groups who tend to be underrepresented in EPI programmes, ${ }^{16}$ such as women, people who are older at first onset of symptoms and recent migrant groups, will be more likely to be managed within primary care. We also expect that among people who are referred to specialised services, there will be little ongoing management of physical or mental health concerns in the primary care context.

\section{Study design}

Using the subgroup of patients with first-episode psychosis in the EMRALD database who are diagnosed in primary care, we will conduct a prospective cohort study of primary care contacts for the 12-month period after the index diagnosis of psychosis.

\section{Outcome measures}

Data will be extracted from the EMRALD database on frequency of contacts, prescription of psychotropic medication and referral to specialised care.

\section{Analysis}

To identify common practise patterns, we will use clustering techniques from the machine-learning literature to identify management practises that tend to co-occur within the population of family physicians (e.g. perhaps psychotropic medication and referral to specialist are often used separately and seldom used together), as well as practise patterns that are seldom or never used. We will also use a bi-clustering technique (sometimes called co-clustering or block clustering ${ }^{40}$ to simultaneously identify subgroups of family physicians who have similar practise patterns based on available data, and subgroups of patients more likely to be managed in primary care.

\section{Sample size}

As described in the previous section, we expect our sample to include approximately 750 cases diagnosed in primary care. This sample size is sufficient for the machine-learning analyses, where the general criterion is that at least ten cases are needed per variable considered in the bi-clustering procedure.

\section{Outputs}

These analyses will provide information on the practise patterns of family physicians when managing first-episode psychosis in primary care, whether particular subgroups of patients are more likely to be receiving ongoing primary care and whether subgroups of family physicians may require additional supports to manage these patients.

\section{Qualitative methods}

\section{Research questions}

The research questions will be driven by the results of the quantitative analyses, and will seek explanations for observed trends. We will additionally address the following research questions: What are the experiences of young people with first-episode psychosis in seeking help from primary care for their early symptoms of psychosis? What do they identify as facilitators or barriers to help-seeking from their family physician? What are the experiences of family physicians in recognising and diagnosing early psychosis in primary care? What supports and resources do family physicians require to manage cases of first-episode psychosis? What do family physicians see as their role in caring for patients with first-episode psychosis after they have been referred and accepted into an EPI programme? And finally, what do intake workers and clinicians at EPI programmes identify as barriers and opportunities for providing collaborative care with family physicians?

\section{Qualitative approach}

We will use a qualitative descriptive approach, which is aimed at producing a comprehensive summary of the phenomenon. ${ }^{41}$ This approach was chosen for the proposed analysis because it is particularly suited to mixed-methods designs and is relevant in health services research for improving the potential utility and uptake of the findings. ${ }^{41,42}$

\section{Sampling}

Maximum variation sampling will be used to select clients from participating EPI sites by gender (demographic variation) and whether they were referred by a family physician (phenomenal variation). ${ }^{43}$ For the family physician interviews, we will approach primary care physicians from the professional networks of study team members. Maximum variation sampling will again be used to select family physicians by gender, years since graduation, practice model, and urban versus rural practice location. Finally, we will conduct one focus group per participating EPI site $(n=6)$, consisting of a range of clinicians, including intake coordinators, case managers and psychiatrists.

\section{Data collection}

Data will be collected by semistructured in-depth interviews, which use an open-ended, conversational technique that focuses on the participants' experience and understanding of an event. ${ }^{44}$ The main interview questions will be developed $a$ priori, based on findings from the quantitative analyses, and incorporated into interview guides that will be used as an outline and to probe areas of interest. ${ }^{42}$ All participants will be asked a set of core questions informed from the quantitative data to identify recurring themes in qualitative responses to specific inquiries. Three versions of the interview guide will be developed and tailored to the stakeholder being interviewed. Interviews will be conducted by telephone for clients and family physicians, which has been shown to yield similar findings to face-to-face interviews, ${ }^{45}$ and via focus group for EPI clinicians. All participants will receive a token for their contribution in the form of gift cards for clients, cash for physicians and lunch for EPI clinicians.

\section{Analysis}

Thematic analysis is the recommended technique for qualitative descriptive studies, as it is less interpretive and focuses on summarising the findings with data-derived codes or themes. ${ }^{41} \mathrm{We}$ will use conventional content analysis, which involves reducing the data into smaller segments or codes, based on the concepts that are represented, and then grouping the material based on shared concepts. ${ }^{46}$ Initial coding will be undertaken individually by three members of the research team. Preliminary interpretations of the qualitative data will then be compared and contrasted, and discussed thoroughly until consensus in the coding structure is achieved. The trustworthiness and validation of preliminary findings will be 
established through this process of systematic, team-based data analysis. Further, the emergent codes will also be refined and modified over the course of the analytic process by the larger research team as new insights are gained that require re-examination of the data.

\section{Sample size}

We expect to recruit 20-30 young people with first-episode psychosis (four or five per site) and 20-30 physicians, based on previous studies that used a qualitative descriptive approach. ${ }^{42}$ We will conduct one focus group at each of the six participating sites, and will aim to include six to ten clinicians per focus group. These are estimated sample sizes, as sampling will occur until maximum variation has been obtained and until the data reaches saturation, which is the point when no additional insights are gained from further data collection efforts. ${ }^{47}$

\section{Outputs}

Interviews with clients will provide descriptions of the motivations and experiences of seeking or not seeking help for psychosis in primary care. Interviews with family physicians will yield in-depth knowledge of their comfort level in recognising and diagnosing psychosis in primary care, and the support required to improve capacity and collaborations with psychiatry. Qualitative focus groups with EPI clinicians will allow us to identify challenges and opportunities for improving transitions of care and collaborations between primary care and EPI programmes.

\section{Discussion}

There is a lack of research on the role of the family physician in EPI. Individually, family physicians may only see a small number of cases of early psychosis per year, but collectively they represent an important access point for psychiatric care. We are proposing to harness the power of this collective by using population-based health administrative data, fully linkable to EMRs from primary care. This will allow us to compile a sufficient sample size to look at actual patterns of health services provision in this population. This would be infeasible with primary data collection strategies, given the low incidence of psychosis. Additionally, we are proposing to use a novel application of machine-learning strategies for data analysis, which will allow us to find complex patterns and trajectories of service use within the rich clinical data of the EMRs. The proposed study will fill a crucial gap in knowledge on how young people with first-episode psychosis interact with the primary care system when seeking help for the early symptoms of psychosis, as well as the practise patterns of family physicians when treating suspected and confirmed cases. Importantly, this project will incorporate the perspectives of young people with first-episode psychosis, family physicians and clinicians at EPI programmes to obtain an indepth understanding of the role of primary care in EPI.

The primary care system is the most widely used service for mental health problems in Canada, ${ }^{48}$ and young people with firstepisode psychosis would benefit from strengthened collaborations between primary care and EPI services. This is particularly relevant for people in rural and remote areas, where a lack of psychiatrists necessitates a greater involvement of family physicians in mental healthcare. ${ }^{49}$ Increased help-seeking in primary care in the period leading up to the first diagnosis of psychosis ${ }^{22}$ presents an opportunity for earlier detection and initiation of treatment, provided that primary care physicians have the practical knowledge for case recognition and the health service context allows for rapid access to specialised treatment, when needed. Clinicians encountering these patients in primary care need to feel confident in their capacity to screen, identify and refer suspected cases of first-episode psychosis. ${ }^{50}$ The outputs from the proposed project will allow for the development of interventions aimed at better supporting family physicians in their central role in pathways to care for firstepisode psychosis. This research will provide essential information on how we can most efficiently use existing resources to build linkages between primary care and EPI services to improve collaboration and continuity of care for young people with first-episode psychosis.

Kelly K. Anderson, Assistant Professor, Department of Epidemiology \& Biostatistics, Schulich School of Medicine \& Dentistry, The University of Western Ontario and Department of Psychiatry, Schulich School of Medicine \& Dentistry, The University of Western Ontario and Mental Health and Addictions Group, Institute for Clinical Evaluative Sciences, Canada; Suzanne Archie, Associate Professor, Department of Psychiatry and Behavioural Neurosciences, McMaster University, Canada; Richard G. Booth Assistant Professor, Arthur Labatt Family School of Nursing The University of Western Ontario,

Panada, Chiachen Cheng Assistant

Canada; Chiachen Cheng, Assistant Professor, Department of Psychiatry, Northern Ontario School of Medicine, Canada; Daniel Lizotte, Assistant Professor, Department of Epidemiology \& Biostatistics, Schulich School of Medicine \& Dentistry, The University of Western Ontario and Department of Computer Science, The University of Western Ontario, Canada; Arlene G. MacDougall, Assistant Professor, Department of Epidemiology \& Biostatistics, Schulich School of Medicine \& Dentistry, The University of Western Ontario and Department of Psychiatry, Schulich School of Medicine \& Dentistry, The University of Western Ontario, Canada; Ross M. G. Norman, Professor Emeritus, Department of Epidemiology \& Biostatistics, Schulich School of Medicine \& Dentistry, The University of Western Ontario and Department of Psychiatry, Schulich School of Medicine \& Dentistry, The University of Western Ontario, Canada; Bridget L. Ryan, Assistant Professor, Department of Epidemiology \& Biostatistics, Schulich School of Medicine \& Dentistry, The University of Western Ontario and Centre for Studies in Family Medicine, Schulich School of Medicine \& Dentistry, The University of Western Ontario, Canada; Amanda L. Terry, Assistant Professor, Department of Epidemiology \& Biostatistics,

Schulich School of Medicine \& Dentistry, The University of Western Ontario and Centre for Studies in Family Medicine, Schulich School of Medicine \& Dentistry, The University of Western Ontario, Canada; Rebecca Rodrigues, Project Coordinator, Department of Epidemiology \& Biostatistics, Schulich School of Medicine \& Dentistry, The University of Western Ontario, Canada

Correspondence: Kelly K. Anderson, Department of Epidemiology \& Biostatistics, The University of Western Ontario, Room K213, Kresge Building, 1151 Richmond Street, London, ON N6A 5C1, Canada. Email: kelly.anderson@schulich.uwo.ca

First received 15 May 2018, final revision 12 Sep 2018, accepted 8 Oct 2018

\section{Funding}

This study is funded by a project grant from the Canadian Institutes of Health Research (PJT 153022) and an Early Researcher Award from the Ontario Ministry of Research, Innovation, and Science (to K.K.A.)

\section{Acknowledgements}

The project co-investigators include Kelly K. Anderson (The University of Western Ontario), Suzanne Archie (McMaster University), Richard G. Booth (The University of Western Ontario), Elisa Candido (Institute for Clinical Evaluative Sciences), Chiachen Chang (Northern Ontario Medical School - Thunder Bay), Saadia Hameed (The University of Western Ontario), Liisa Jaakkimainen (Institute for Clinical Evaluative Sciences), Paul Kurdyak (Centre for Addiction and Mental Heath) Dar (The University of Western Ontario), Ross Norman (The University of Western Ontario), Lena Palaniyappan (The University of Western Ontario), Rebecca Rodrigues (The University of Western Ontario), Bridget L. Ryan (The University of Western Ontario), Amanda L. Terry (The University of Western Ontario), and Aristotle Voineskos (Centre for Addiction and Mental Health).

\section{References}

1 Marshall M, Lewis S, Lockwood A, Drake R, Jones $\mathrm{P}$, Croudace T. Association between duration of untreated psychosis and outcome in cohorts of firstepisode patients: a systematic review. Arch Gen Psychiatry 2005; 62: 975-83.

2 Perkins DO, Gu H, Boteva K, Lieberman JA. Relationship between duration of untreated psychosis and outcome in first-episode schizophrenia: a critical review and meta-analysis. Am J Psychiatry 2005; 162: 1785-804.

3 Harrison G. Recovery from psychotic illness: a 15- and 25-year international follow-up study. Br J Psychiatry 2001; 178: 506-17.

4 Ontario Ministry of Health and Long Term Care. Program Policy Framework for Early Intervention in Psychosis. Government of Ontario, 2004. 
5 Anderson KK, Fuhrer R, Malla AK. The pathways to mental health care of first-episode psychosis patients: a systematic review. Psychol Med 2010; 40 1585-97.

6 Goldberg D, Huxley P. Models for mental illness. In Common Mental Disorders: A Bio-Social Model: 1-52. Tavistock Routledge, 1992.

7 Kessler RC, Berglund P, Demler O, Jin R, Merikangas KR, Walters EE. Lifetime prevalence and age-of-onset distributions of DSM-IV disorders in the National Comorbidity Survey Replication. Arch Gen Psychiatry 2005; 62: 593-602.

8 Wang PS, Angermeyer M, Borges G, Bruffaerts R, Tat Chiu W, DE Girolamo G, et al. Delay and failure in treatment seeking after first onset of mental disorders in the World Health Organization's World Mental Health Survey Initiative. World Psychiatry 2007; 6: 177-85

9 Anderson KK, Fuhrer R, Schmitz N, Malla AK. Determinants of negative pathways to care and their impact on service disengagement in first-episode psychosis. Soc Psychiatry Psychiatr Epidemiol 2013; 48: 125-36.

10 Flora N, Anderson KK, Ferrari M, Tuck A, Archie S, Kidd S, et al. Comparative analysis of pathways to early intervention services and duration of untreated psychosis in two Canadian cities. Early Interv Psychiatry 2017; 11: 517-21.

11 Anderson KK, Flora N, Ferrari M, Tuck A, Archie S, Kidd S, et al. Pathways to first-episode care for psychosis in African-, Caribbean-, and European-origin groups in Ontario. Can J Psychiatry 2015; 60: 223-31.

12 Archie S, Akhtar-Danesh N, Norman R, Malla A, Roy P, Zipursky RB. Ethnic diversity and pathways to care for a first episode of psychosis in Ontario. Schizophr Bull 2010; 36: 688-701.

13 Anderson KK, Fuhrer R, Malla AK. 'There are too many steps before you get to where you need to be': help-seeking by patients with first-episode psychosis. $J$ Ment Heal 2013; 22: 384-95.

14 Anderson KK, Kurdyak P. Factors associated with timely physician follow-up after a first diagnosis of psychotic disorder. Can J Psychiatry 2017; 62: 268-77.

15 Anderson KK, Fuhrer R, Wynant W, Abrahamowicz M, Buckeridge DL, Malla A Patterns of health services use prior to a first diagnosis of psychosis: the importance of primary care. Soc Psychiatry Psychiatr Epidemiol 2013; 48 1389-98.

16 Anderson KK, Norman R, MacDougall AG, Edwards J, Palaniyappan L, Lau C, et al. Disparities in access to early psychosis intervention services: comparison of service users and nonusers in health administrative data. Can J Psychiatry 2018; 63: 395-403.

17 El-adl M, Burke J, Little K. First-episode psychosis: primary care experience and implications for service development. Psychiatr Bull 2009; 33: 165-8.

18 Renwick L, Gavin B, McGlade N, Lacey P, Goggins R, Jackson D, et al. Early intervention service for psychosis: views from primary care. Early Interv Psychiatry 2008; 2: 285-90.

19 Simon AE, Lauber C, Ludewig K, Braun-Scharm H, Umbricht DS. General practitioners and schizophrenia: results from a Swiss survey. Br J Psychiatry 2005; 187: $274-81$.

20 Simon AE, Lester $\mathrm{H}$, Tait L, Stip E, Roy P, Conrad G, et al. The International Study on General Practitioners and Early Psychosis (IGPS). Schizophr Res 2009; 108 182-90.

21 Giannopoulos P, Carroll A, Ebmeier KP. Improving the detection and treatment of schizophrenia. Practitioner 2014; 258(2): 11-6.

22 Nørgaard $\mathrm{H}$, Søndergaard Pedersen $\mathrm{H}$, Fenger-Grøn M, Mors O, Nordentoft M, Vestergaard $M$, et al. Increased use of primary care during 6 years of prodromal schizophrenia. Acta Psychiatr Scand 2016; 134: 225-33.

23 Yung AR, McGorry PD. The prodromal phase of first-episode psychosis: past and current conceptualizations. Schizophr Bull 1996; 22: 353-70.

24 Lester H, Tritter JQ, Sorohan H. Patients' and health professionals' views on primary care for people with serious mental illness: focus group study. BMJ 2005 330: 1122

25 Verdoux H, Cougnard A, Grolleau S, Besson R, Delcroix F. How do general practitioners manage subjects with early schizophrenia and collaborate with mental health professionals? A postal survey in south-western France. Soc Psychiatry Psychiatr Epidemiol 2005; 40: 892-8.

26 Gavin B, Cullen W, O'Donoghue B, Ascencio-Lane JC, Bury G, O'Callaghan E. First episode schizophrenia in general practice: a national survey. Ir 」 Psychol Med 2006; 23: 6-9.

27 Anderson KK, Norman R, MacDougall A, Edwards J, Palaniyappan L, Lau C, et al. Effectiveness of early psychosis intervention: comparison of service users and nonusers in population-based health administrative data. Am J Psychiatry 2018; 175: 443-52.

28 Shiers D, Lester H. Early intervention for first episode psychosis. BMJ 2004; 328 : 1451-2.

29 Reynolds N, Wuyts P, Badger S, Fusar-Poli P, McGuire P, Valmaggia L. The impact of delivering GP training on the clinical high risk and first-episode psychosis on referrals and pathways to care. Early Interv Psychiatry 2015; 9: 459-66.

30 Power $\mathrm{P}$, lacoponi E, Reynolds N, Fisher H, Russell M, Garety P, et al. The Lambeth Early Onset Crisis Assessment Team Study: general practitioner education and access to an early detection team in first-episode psychosis. $\mathrm{Br} \mathrm{J}$ Psychiatry 2007; 191: 3-10.

31 Lester $\mathrm{H}$, Birchwood M, Freemantle N, Michail M, Tait L. REDIRECT: cluster randomised controlled trial of GP training in first-episode psychosis. Br J Gen Pract 2009; 59: e183-90.

32 Lloyd-Evans B, Crosby M, Stockton S, Pilling S, Hobbs L, Hinton M, et al. Initiatives to shorten duration of untreated psychosis: systematic review. $\mathrm{Br} J$ Psychiatry 2011; 198: 256-63.

33 Perez J, Jin H, Russo DA, Stochl J, Painter M, Shelley G, et al. Clinical effectiveness and cost-effectiveness of tailored intensive liaison between primary and secondary care to identify individuals at risk of a first psychotic illness (the LEGs study): a cluster-randomised controlled trial. Lancet Psychiatry 2015; 2(11): 984-93.

34 Creswell JW, Plano Clark VL. Designing and Conducting Mixed Methods Research. Sage Publications, 2007.

35 Kurdyak P, Lin E, Green D, Vigod S. Validation of a population-based algorithm to detect chronic psychotic illness. Can J Psychiatry 2015; 60: 362-8.

36 Shah BR, Hux JE, Laupacis A, Zinman B, Cauch-Dudek K, Booth GL. Administrative data algorithms can describe ambulatory physician utilization. Health Serv Res 2007; 42: 1783-96.

37 Bradford DW, Kim MM, Braxton LE, Marx CE, Butterfield M, Elbogen EB. Access to medical care among persons with psychotic and major affective disorders. Psychiatr Serv 2008; 59: 847-52.

38 Mitchell T. Machine Learning. McGraw Hill, 1997.

39 Yung AR, Nelson B, Stanford C, Simmons MB, Cosgrave EM, Killackey E, et al. Validation of 'prodromal' criteria to detect individuals at ultra high risk of psychosis: 2 year follow-up. Schizophr Res 2008; 105: 10-7.

40 Zhao H, Wee-Chung Liew A, Wang DZ, Yan H. Biclustering analysis for pattern discovery: current techniques, comparative studies and applications. Curr Bioinform 2012; 7: 43-55(13).

41 Sandelowski M. Focus on research methods whatever happened to qualitative description? Res Nurs Health 2000; 23: 246-55

42 Sullivan-Bolyai S, Bova C, Harper D. Developing and refining interventions in persons with health disparities: the use of qualitative description. Nurs Outlook 2010; 53: 127-33.

43 Patton MQ. Qualitative Research and Evaluation Methods, 2nd edn: 145-98. Sage Publications, 1990

44 Miller WL, Crabtree BF. Depth interviewing. In Doing Qualitative Research, 2nd edn (eds BF Crabtree, WL Miller): 89-107. Sage Publications, 1999.

45 Sturges JE, Hanrahan KJ. Comparing telephone and face-to-face qualitative interviewing: a research note. Qual Res 2004; 4: 107-18.

$46 \mathrm{Hsieh} \mathrm{H-F}$, Shannon SE. Three approaches to qualitative content analysis. Qual Health Res 2005; 15: 1277-88.

47 Morse JM. The significance of saturation. Qual Health Res 1995; 5: 147-9.

48 Vasiliadis H-M, Lesage A, Adair C, Boyer R. Service use for mental health reasons: cross-provincial differences in rates, determinants, and equity of access. Can J Psychiatry 2005; 50: 614-9.

49 Kurdyak P, Stukel TA, Goldbloom D, Kopp A, Zagorski BM, Mulsant BH. Universal coverage without universal access : a study of psychiatrist supply and practice patterns in Ontario. Open Med 2014; 8: e81-93.

50 Boydell KM, Gladstone BM, Volpe T. Understanding help seeking delay in the prodrome to first episode psychosis: a secondary analysis of the perspectives of young people. Psychiatr Rehabil J 2006; 30: 54-60. 